\title{
Desarrollo conceptual de los factores críticos de éxito en la adquisición y fusión de empresas: contexto para un estudio de organizaciones mexicanas (Conceptual development of critical success factors in the acquisition and merger of enterprises: context for a study of Mexican organizations)
}

\author{
Alejandro Rodríguez Miechielsen, Gustavo Juan Alarcón Martínez \& \\ Joel Mendoza Gómez \\ Universidad Autónoma de Nuevo León, Facultad de Contaduría Pública y Administración, \\ San Nicolás de los Garza, N.L., México. \\ Email: alejandro.rodriguez@sinergiadeportiva.mx
}

Keywords: acquisition, critical success factors, merger

\begin{abstract}
This paper presents a conceptual development on the variables that make up the processes of acquisition and merger holding companies in pursuit of greater strategic value creation. From research questions, is reviewed in detail each of the variables of these processes, in this manner, it is possible to raise further study to identify the relative importance of each of these variables. We propose a graphical model that integrates the conceptual development of this work.
\end{abstract}

Palabras clave: adquisición, factores críticos de éxito, fusión.

Resumen: En este trabajo se presenta un desarrollo conceptual sobre las variables que integran los procesos de adquisición y fusión que llevan a cabo las empresas en la búsqueda estratégica de mayor creación de valor. De acuerdo a preguntas de investigación, se revisa con detalle cada una de las variables de los mencionados procesos; de esta manera, se plantea, para un posterior estudio empírico, la identificación de las variables en cuestión. Finalmente, se propone un modelo gráfico que integra el desarrollo de este trabajo conceptual. 


\section{Introducción.}

Las empresas en la actualidad están obligadas a permanecer alertas, mediante la observación, no sólo de lo que el mercado demanda, sino también de la identificación de cuáles son las empresas competidoras listas para satisfacer dichas demandas y otras nuevas. En el mundo globalizado, un factor clave de las empresas es la capacidad para ofrecer atractivos productos y servicios, así como, de la eficiencia para atender a los distintos mercados con sus particulares características geográficas y culturales. La competencia obliga a operar con economías de escala, buscar nuevos mercados e integrar líneas de producción. Esto se puede lograr a través de diversas estrategias, entre ellas, la adquisición y fusión (A\&F) de empresas, estrategia que es el motivo central en el desarrollo de este estudio conceptual.

En los países latinoamericanos, Martínez (2012) menciona que la alternativa de realizar A\&F se ha incrementado en el último trienio; entre los años 2009 y 2011 , aumentó de $2.3 \%$ a $5 \%$ el total acumulado mundial. El crecimiento de las A\&F se debe a que es considerada una buena fórmula de agregar productos, mercados, capacidades varias, fuentes alternas de capital y financiamiento y además de reducir el riesgo de pérdidas. La crisis financiera de 2008 afectó la actividad económica en cuanto a A\&F. En 2009, el efecto fue negativo. En el 2010 fue positivo, durante este año, el nivel de las A\&F realizadas en México fue importante, tanto en pesos como en volumen, reflejando la confianza de los inversionistas. El 2011 fue considerado como un período de estabilización de dicho fenómeno (Hernández \& Silva, 2011).

Dado que las A\&F han cobrado gran importancia, es fundamental tomar en cuenta las oportunidades y los riesgos que ello conlleva. Al respecto, es particularmente conveniente analizar los Factores Críticos de Éxito (FCE) en los procesos de A\&F de empresas, para conocer la importancia relativa de los mismos. En relación a un estudio de este tipo, a continuación se presenta inicialmente el planteamiento del problema, el cual incluye las preguntas y los objetivos de la investigación. Posteriormente, se desarrolla la revisión de literatura sobre el particular, para fundamentar el desarrollo conceptual. Por último, se presenta el modelo conceptual propuesto, así como las conclusiones de este trabajo. 


\section{Planteamiento del problema.}

En un contexto global, las A\&F han tenido diversos grados de éxito desde la década de los años 60 al presente. En las décadas de los años 60 y 70 , las empresas con ganancias por encima de la media antes de la adquisición obtuvieron caídas en sus beneficios tras la operación, debido a la pérdida del control de gestión (Ravenscraft, 1983; en Zozaya, 2007, P.12).

En dicha época, las A\&F representaban estrategias de alto riesgo con bajo porcentaje de éxito. Con la finalidad de mejorar esta situación, la mayoría de los estudios sobre A\&F realizados en las décadas de los 80 y los 90 desde diferentes perspectivas, han tratado de explicar las razones de los resultados obtenidos por las empresas tras las operaciones de A\&F, enfocándose en la búsqueda de elementos clave para lograr el éxito. El ajuste estratégico, el organizativo y el cultural fueron los factores de estudio asociados al mejoramiento de los resultados de las adquisiciones (Chatterjee, 1986; Jemison, D.B. \& Sitkin, S.B, 1986; Pablo, 1994).

Para los años 90, la mayoría de las A\&F se relacionaban con empresas estadounidenses; sin embargo, una década después, estas operaciones crecieron, ocurriendo también en Europa, como consecuencia de la globalización, la incorporación de nuevos países a la Unión Europea, la eliminación de barreras a la inversión extranjera y los cambios regulatorios sobre el gobierno corporativo generados tras los escándalos de empresas como Enron, Parmalat o WorldCom (Feito \& Menéndez, 2011).

El éxito de las A\&F, para Morck, Schleifer \& Vishny (1990) se ha relacionado de manera significativa, con altos niveles de rentabilidad previos de la fusión de las empresas adquirientes. Otros estudios realizados (Sherer \& Ross, 1990; Morck et al., 1990), coinciden en que las A\&F que involucran a empresas que pertenecen a industrias relacionadas tienden a tener un mayor éxito en comparación con el caso de empresas no relacionadas.

Rovit \& Lemire (2003) señalan que las empresas que acumulan experiencia en A\&F se vuelven diestras en manejar estos procesos y logran mejores resultados. Dentro de este grupo, en México se encuentra la empresa cementera Cemex, la cual, mediante estrategias de adquisición horizontal, ha realizado adquisiciones de empresas relacionadas. Según el sitio web de la empresa www.cemex.com, su estrategia se ha dividido en 
cuatro etapas: expansión nacional, exportación, proceso de crecimiento trasnacional y diversificación funcional.

En cambio, el fracaso de muchas fusiones, Moyer (2004) lo atribuye a un proceso deficiente de integración. En general, se observa una falta de consenso, entre los académicos/investigadores, respecto al rol de los FCE dentro de los procesos de adquisición y fusión.

De acuerdo con McCiurkan \& Torres (2011), en México se anunciaron transacciones por 16 billones de dólares para el año 2010, una cifra mayor al promedio de los últimos diez años, que fue de 11 mil 300 millones. Las estimaciones para 2011 sugieren que es buen momento para adentrarse en los procesos estructurados de A\&F.

Según Hernández \& Silva (2011), durante el 2011 una sola transacción en México, la adquisición de Femsa Cerveza SA de CV por la holandesa Heineken NV, representó siete mil 691 millones de dólares y, por mucho, fue la operación más importante del 2011 sucedida en México. Otra que destacó por su tamaño fue la adquisición del restante $24.9 \%$ de Grupo Financiero Santander SA de CV a Bank of America Corporation, por parte de Banco Santander SA.

Es conveniente señalar, en este momento, la distinción que en este artículo se hace entre adquisición y fusión. Ambos son procesos que conllevan la búsqueda de un resultado positivo para la empresa que de manera proactiva los lleva a cabo. Por una parte, existe el proceso de adquisición que consiste en llevar a cabo una serie de evaluaciones que permiten establecer si la empresa adquirida es compatible con la empresa adquiriente, de manera tal que si existe la compatibilidad, se toma la decisión de adquirirla permitiendo calificarla como una adquisición exitosa.

Por otra parte, el proceso de fusión, también consiste en una serie de evaluaciones que permiten establecer si el resultado de la adquisición generó la integración y las sinergias esperadas por la empresa adquiriente, de tal manera que pueda considerarse un éxito la fusión de ambas empresas llevada a cabo con anterioridad.

De esta manera, el proceso completo de evaluación de las A\&F incorpora un horizonte de tiempo en dos períodos, uno para cada uno de los procesos antes mencionados, lo cual implica que varios de los elementos estudiados o evaluados, considerados como factores de éxito, en dichos procesos aparezcan en ambos períodos y formen parte de los factores a 
estudiar en este desarrollo conceptual. Así, una vez que se hace la evaluación correspondiente en cada etapa puede considerarse completo el proceso de A\&F.

Dentro de los elementos que caracterizan el éxito del proceso de adquisición se pueden mencionar los siguientes: la toma de decisiones para llevar a cabo la adquisición, que incorpora una información adecuada de los elementos fundamentales que se evaluaron en las organizaciones participantes los cuales incluyen los elementos relacionados con el mercado producto, con la operación, las finanzas y el capital humano, así como, el diagnóstico inicial de vinculación entre ambas empresas. Así, es interesante conocer los factores clave y su importancia en este proceso de evaluación.

En lo relativo a los elementos que caracterizan el éxito del proceso de fusión se pueden mencionar los siguientes: una evaluación de la integración y de la sinergia que se generan en el mercado - producto, considerando la operación, las finanzas y el capital humano, así como el aseguramiento legal y contable entre ambas empresas. De la misma manera, en este proceso se requiere identificar la importancia relativa de estos factores.

Los dos anteriores procesos deben considerarse como tales, para no perder de vista el resultado final que se busca en las A\&F.

Cabe señalar que además de la revisión de literatura para identificar a estos factores de éxito, se encuentra la experiencia de más de 30 años del primer autor de este trabajo, quien ha llevado a cabo diferentes A\&F; asimismo, se ha contado con información proveniente de entrevistas cualitativas con expertos en la materia. De esta manera, en el proceso de adquisición, de acuerdo con la información acopiada, en este estudio conceptual se han identificado como relevantes los siguientes factores:

1.- La investigación y diagnóstico integral (Due dilligence) el cual se refiere a realizar detalladamente una serie de pequeñas auditorías sobre aspectos financieros, fiscales, de recursos humanos, sindicales, legales $y$ operativos de una empresa.

2.- El acoplamiento estratégico de producto-mercado, que consiste en adecuar la cartera de productos de la empresa adquirida a los objetivos y estrategia de la empresa adquiriente para ser competitiva y abarcar mayor parte del mercado en el que desarrolla su actividad. 
3.- El acoplamiento operativo que implica integrar los procesos, las líneas de producción, la tecnología, las patentes, complementarse en recursos entre ambas empresas; por un lado la adquirida, y por otra, la adquiriente.

4.- El acoplamiento financiero, que tiene que ver con adaptar los sistemas financieros de la empresa adquirida con los sistemas financieros de la empresa adquiriente, con la finalidad de hacer eficiente la administración del dinero y maximizar el valor de la empresa.

5.- El acoplamiento de capital humano, el cual está relacionado con administrar las capacidades, actitudes, destrezas y conocimientos de cada una de las personas de ambas empresas involucradas en el proceso de adquisición, con el propósito de optimizar sus labores productivas.

De igual manera, tomando en cuenta la información mencionada previamente, se identificaron en el proceso de fusión los siguientes aspectos:

1.- El aseguramiento legal y contable, aspecto con el cual se busca confirmar la situación legal de la empresa objeto de la operación, la identificación de los riesgos asociados y las posibles contingencias en materia legal y contable.

2.- La sinergia producto-mercado, que se refiere según Manso (2003) a las distintas combinaciones producto-mercado que se definen como unidades estratégicas de negocio de la empresa; al respecto caben tantos ámbitos como misiones posibles (mercados-objetivo) que la empresa pueda combinar.

3.- La sinergia operativa que se refiere a la colaboración para que la suma de los esfuerzos operativos en conjunto de las empresas involucradas en la operación de fusión sean superiores a la suma de los esfuerzos individuales.

4.- La sinergia financiera que consiste en el aumento del valor de las empresas fusionadas creado por ventajas estrictamente financieras vinculadas a las fusiones y absorciones, que no pueden ser alcanzadas por los inversionistas particulares mediante la gestión privada de su cartera de acciones (Lewellen, 1971).

5. La cultura asimilada representada por el grado en que la cultura organizacional de ambas empresas logran valores compartidos entre sus miembros, aspecto que distingue a una organización de otras (Robbins, 1999). 
Los anteriores factores que conllevan al éxito en los procesos de A\&F siguen siendo discutidos por parte de diversos estudiosos del tema, sin que se haya logrado establecer el grado de importancia que representa cada uno de ellos para el éxito en las A\&F respecto a los demás. Adicionalmente cabe mencionar que, estos elementos no se tratan en estos procesos de manera conjunta o integral.

En nuestro contexto, de acuerdo a De los Ríos (2007), en México, solamente se cuenta con información marginal y secundaria sobre el tema. De la revisión de la literatura, se encontró que las investigaciones que abordan los estudios, desde un punto de vista integral o desde una perspectiva cuantitativa, sobre adquisiciones, fusiones, absorciones, toma de control y alianzas, son insuficientes; además, diversos estudios señalan que la tasa de fracaso de las A\&F varía entre el $70 \%$ y $90 \%$ según Christensen, Alton, Rising, \& Walswck (2011).

Los estudios analizados aportan luces sobre algunos factores que se consideran determinantes para el éxito; sin embargo, no consideran los posibles factores de manera conjunta y no los jerarquizan. Resulta necesario analizar los FCE y el grado de importancia que representa cada uno de ellos respecto a los demás; ello bajo una perspectiva integral, considerando por una parte los FCE de las Adquisiciones y por la otra los FCE de las Fusiones, ya que implican procesos distintos, aunque sería deseable realizarlos, a la vez, sobre la base de escenarios.

De acuerdo a lo planteado con anterioridad las preguntas de investigación para el estudio en cuestión son las siguientes:

¿Es posible identificar la importancia relativa de cada uno de los factores de un proceso de adquisición exitosa, como son: la investigación y diagnóstico integral (Due dilligence), el acoplamiento producto-mercado, el acoplamiento operativo, el acoplamiento financiero y el acoplamiento de capital humano?

¿Es posible identificar la importancia relativa de cada uno de los factores de un proceso de fusión exitosa, como son: el aseguramiento legal y contable, la sinergia producto-mercado, la sinergia operativa, la sinergia financiera y la cultura asimilada? 
De estas preguntas se deriva el siguiente objetivo:

Desarrollar un modelo de análisis de los procesos de adquisición y fusión de empresas, en el que se identifique la importancia relativa de cada uno de los factores incorporados en dichos procesos.

De esta manera, a continuación se presenta la revisión de la literatura que sustenta el desarrollo de este modelo conceptual.

\section{Revisión de literatura.}

Perspectivas teóricas en el estudio de las A\&F

En este apartado, se presentan algunas perspectivas teóricas con las que se ha intentado explicar motivaciones, premisas y elementos relacionados con las A\&F. Posteriormente se presenta el desarrollo de cada una de las variables que integran el modelo de análisis que se propone en este trabajo para el estudio de los procesos de las A\&F

El estudio de los procesos de A\&F se ha llevado a cabo bajo distintas perspectivas teóricas, las cuales destacan diversos elementos de dichos procesos, o bien se refieren al resultado final que la empresa busca alcanzar. Así, encontramos por una parte, en la teoría del ciclo de vida (Mueller, 1972), que las A\&F se llevan a cabo para modificar, alterar o reforzar alguna etapa del ciclo de vida de una empresa. Asimismo en esta perspectiva, se destaca que los directivos de la empresa buscan su beneficio; de esta manera surge la hipótesis teórica de lograr un máximo de crecimiento (Mueller, 1972) a través de la evaluación del acoplamiento estratégico de producto-mercado.

La previsión del acoplamiento humano, es otra de las perspectivas teóricas utilizadas en el estudio de las A\&F. A este respecto se consideran los siguientes aspectos: la presencia de un alto grado de ansiedad en este proceso (Seo \& Hill, 2005) que deriva en la búsqueda de una posición positiva de su propio grupo (Seo \& Hill, 2005) la presentación de los aspectos de justicia para el personal involucrado, aspecto que se estudia bajo la perspectiva de la justicia organizacional (Seo \& Hill, 2005).

También en relación al comportamiento del capital humano, la cultura asimilada es otro de los aspectos que se considera en el análisis de las A\&F, al respecto destaca lo siguiente: la importancia de resolver los choques 
culturales del capital humano de ambas empresas en una perspectiva de aculturación (Seo \& Hill, 2005); otro aspecto a considerar es el relacionado con las características del trabajo y el rediseño de los puestos de trabajo para lograr una mayor integración del capital humano (Seo \& Hill, 2005).

Otra perspectiva teórica que se ha utilizado con frecuencia en el estudio de las A\&F, es la financiera. Al respecto se han estudiado los siguientes aspectos identificados también con el momento o momentum de la toma de decisión: en una perspectiva neoclásica, se asume que los administradores actúan con base en el propósito de incrementar el valor de los accionistas debido al aumento de las sinergias derivadas del proceso de A\&F (Rosen, 2006); en relación al acoplamiento financiero uno de los enfoques enfatiza las creencias optimistas, las cuales están relacionadas a la reacción del mercado a los anuncios de A\&F, que promueven un optimismo que se refleja en el precio de las acciones en el corto plazo, sin embargo habría que considerar que, en el largo plazo dicho precio regresa a su normalidad (Rosen, 2006).

En cuanto las sinergias financieras de las A\&F una postura teórica es aquella que considera la fusión como una desinversión (Fluck \& Lynch, 1999); esta perspectiva financiera enfatiza la importancia de evaluar la capacidad del proyecto en vinculación con su supervivencia. Otros enfoques enfatizan la necesidad de considerar la eficiencia en la sinergia financiera (Trautwein, 1990).

La importancia de desarrollar de manera completa y efectiva una investigación y diagnóstico integral (Due dilligence) ha llevado a otras explicaciones teóricas relacionadas con el papel de los directivos que toman las decisiones. Al respecto se considera que si los directivos tienen una orientación positiva sobre el Due dilligence, este se llevará a cabo de manera completa, en cambio, si la motivación es negativa, porque el directivo busca su propio beneficio, la actividad de diagnóstico no se llevará a cabo de manera efectiva (Trautwein, 1990). Otro aspecto teórico en esta misma línea de argumentación, destaca la racionalidad limitada de los directivos, aspecto que los lleva a considerar pocas alternativas, lo cual puede afectar la actividad de diagnóstico (Due dilligence) en un sentido negativo (Trautwein, 1990).

Una perspectiva teórica adicional relativa al proceso de las A\&F es la relacionada con el aseguramiento contable legal pues se considera 
importante tener información contable que refleje los resultados económicos del proceso de integración (Giner \& Pardo, 2004). En esta misma línea, pero con énfasis en los aspectos legales, se destaca la necesidad de considerar las disposiciones legales relativas a fusiones que pueden derivar en un monopolio (Trautwein, 1990).

En las explicaciones teóricas anteriores, se encuentra por una parte, el énfasis en una presencia significativa de los comportamientos de los directivos y como éstos influyen en los procesos de A\&F; por otra parte, las explicaciones se orientan principalmente a los aspectos financieros y de capital humano. Cabe destacar que existen explicaciones teóricas relacionadas con dichos procesos, las cuales se referirán adelante, relativas a los factores que integran cada uno de los procesos propuestos en este desarrollo conceptual.

\section{Variable Dependiente.}

El éxito en las $A \& F$.

"El éxito en la vida no tiene que ver con la suerte o con estar en el lugar apropiado en el momento oportuno. Tiene que ver con la forma en que realizamos nuestro trabajo a través del tiempo, y nos mantenemos enfocados en una determinada meta por la suficiente cantidad de años". (Andres Panasiuk, 2004)

También se puede definir el éxito, de acuerdo a John R. Wooden \& Carty (2006), como la paz mental que nace como resultado natural de la íntima satisfacción lograda al saber que uno hizo lo mejor que pudo para convertirse en lo mejor que uno es capaz de ser.

Para Luck (1996) una empresa es exitosa si el dueño o administrador ha obtenido un excedente en las ganancias, en el tiempo que se ha propuesto como meta.

De acuerdo a su origen etimológico la palabra éxito proviene del latín exitus, que significa "salida". Representa un concepto subjetivo, ya que dependerá de cada persona, encontrar la salida apropiada hacia el logro de sus metas que lo transforme en exitoso; es decir obtener un resultado final satisfactorio o positivo, aunque la acción no haya superado las expectativas, en otras palabras alcanzar lo que se propuso al principio. 
Como hemos visto y de acuerdo con Ketelhöhn y Marin $(2009$, p.20) no existe una definición concreta para el éxito, ya que algunos académicos, por ejemplo, lo definen como el cambio en las utilidades operativas de la empresa resultante durante un período de tiempo arbitrario. Mientras otros lo hacen basados en la reacción de los precios de las acciones de la compañía resultante en el mercado de valores durante un período de tiempo.

Una vez dada algunas de las diferentes definiciones que la palabra éxito puede tomar, se establece el significado que tendrá "el éxito" en este artículo, por un lado en la adquisición y, por otro, en la fusión; ambos son procesos que conllevan la búsqueda de un resultado positivo para la empresa que de manera proactiva los lleva a cabo.

Por una parte, existe el proceso de adquisición que consiste en llevar a cabo una serie de evaluaciones que permiten establecer si la empresa adquirida es compatible con la empresa adquiriente, de manera tal que si existe la compatibilidad, en el tiempo establecido por la adquiriente, se toma la decisión de adquirirla permitiendo calificarla como una adquisición exitosa.

Por lo tanto, las adquisiciones exitosas, en general, implican la necesidad de contar con metas alcanzables, una estrategia entendible, no pagar un precio alto y la aplicación de un proceso de integración eficiente.

Por otra parte, el proceso de fusión, también consiste en una serie de evaluaciones que permiten establecer si el resultado de la adquisición generó la integración y las sinergias esperadas, en el tiempo establecido, por la empresa adquiriente, de tal manera que pueda considerarse un éxito la fusión de ambas empresas llevada a cabo con anterioridad.

El proceso completo de evaluación de las A\&F incorpora un horizonte de tiempo en dos periodos, uno para el proceso de adquisición y otro relativo a la fusión con elementos que permiten su evaluación. Así, una vez que se hace la evaluación correspondiente en cada etapa puede considerarse completo el proceso de A\&F.

En general el éxito en las A\&F se ha relacionado con diversos factores. Por ejemplo, para Morck, Schleifer \& Vishny (1990) el éxito de las A\&F se ha relacionado, de manera significativa, con altos niveles de rentabilidad previos de la fusión de las empresas adquirientes. 
Rovit \& Lemire (2003) señalan que las empresas que acumulan experiencia en A\&F se vuelven diestras en manejar estos procesos y logran mejores resultados.

De acuerdo a la experiencia del primer autor, con el soporte de los expertos entrevistados, se han definido los factores involucrados en el éxito de las A\&F en dos grupos. Por un lado los factores que caracterizan el éxito en la adquisición, que simbolizan la compatibilidad de la empresa adquiriente con la empresa adquirida, y por otro lado los factores que caracterizan el éxito en la fusión, que simbolizan la generación de sinergias después de haberse concebido la compatibilidad de las empresas involucradas en el proceso de adquisición.

Es decir mientras que en la adquisición se busca la compatibilidad de las empresas involucradas, en la fusión se espera que la compatibilidad dada en la adquisición genere sinergias que agreguen valor a la empresa y contribuya con el logro de metas en el periodo establecido.

Se puede concluir que para alcanzar el éxito es necesario primero fijar bien nuestras metas y luego, a partir de ellas, dirigir nuestras acciones, con confianza en nuestra experiencia y con la participación de un grupo de expertos de cada una de las áreas funcionales de la empresa (considerando el aspecto legal y contable; marketing; operación; financiero; recursos humanos) evaluar la viabilidad de la adquisición y de la fusión.

Ugalde (2009) señala que existen tres razones principales por las cuales las empresas se adquieren y fusionan a otras: 1) mejorar los ingresos, 2) reducir los costos e 3) incrementar sus niveles de conocimiento e innovación. Las combinaciones de negocios explotan sinergias que mejoran los ingresos de las empresas al reinvertir sus recursos (Capron, 1999).

En suma, los elementos que caracterizan el resultado final exitoso de las A\&F, el cual no es visible inmediatamente sino después de un período de tiempo adecuado son, entre otros, los siguientes: el logro de las metas personales de los propietarios de las empresas; el tamaño de la producción; la implementación de nuevas tecnologías, y la penetración de nuevos mercados. Como se puede observar, el éxito puede ser estimado desde diferentes perspectivas adicionales a las financieras tales como las utilidades, el retorno de la inversión, la rotación de activos, etc. 


\section{Variables independientes.}

Investigación y diagnóstico integral (Due dilligence).

Aunque las A\&F se han convertido en una forma de crecimiento muy común utilizada por las empresas, es sorprendente el nivel de fracaso de las mismas. De acuerdo con Hope (2001) operaciones que en su momento fueron espectaculares como las de Synkro y Kayser Roth o Vitro con Anchor Glass ponen de manifiesto que, entre empresas, la gula y la vanidad pueden ser puertas al infierno. Sin embargo, empresas como Cemex, Bimbo, IMSA, Carso, Bachoco, Elektra, por citar unos ejemplos, podrían demostrar justo lo contrario.

La necesidad de conocer a profundidad la empresa que se va a adquirir, implica la necesidad de realizar un diagnóstico integral o un Due dilligence, lo cual conlleva a cabo el siguiente análisis: a) un proceso minucioso de evaluación de todos los aspectos de la empresa a adquirir y fusionar, b) auditorías: financieras, fiscales, de recursos humanos, sindicales y operativas, c) valuación de un tercero (si se requiere), d) inspección de la(s) propiedad(es), e) comprobar escrituras, y f) notificar a agencias gubernamentales (si se requiere).

Un ejemplo de la importancia que tiene el Due dilligence en estos procesos de A\&F, se observa en la transacción Femsa-Heineken. A mediados de 2009 se acercaron los posibles socios a la empresa Femsa con la intención de adquirir. A principios de octubre, el mercado se enteró de las intenciones de venta de Femsa, su acción creció $17 \%$ en un solo día y gracias a la filtración obtuvieron nuevas propuestas, entre las cuales estaba SABMiller. En tres de las cuatro opciones que tenían llegaron a la etapa de Due dilligence. Ganó Heineken la transacción (Anderson \& Ortega, 2010).

En relación al concepto, el término Due dilligence es traducido al español como la "debida diligencia" y tiene como objetivo examinar minuciosamente las áreas más importante de la empresa objetivo que está en proceso de ser adquirida o fusionada con otra empresa.

Según Hoskisson, Hitt \& Ireland (2004, p.251) se puede definir el Due dilligence como el proceso por el cual un adquiriente potencial evalúa una empresa objetivo o sus activos de cara a una adquisición. 
De acuerdo con lo anterior, la definición del Due dilligence o diagnóstico preliminar se concibe como un análisis profundo de la(s) empresa(s) a adquirir. En este sentido, el Due dilligence, implica el análisis y evaluación de todos los elementos clave que inciden en la decisión de proceder con una adquisición. Puede tratarse de una obligación legal, pero el término comúnmente es más aplicable a investigaciones voluntarias.

Un proceso estructurado requiere una serie de estudios que ayuden a confirmar el impacto de la adquisición e integración del negocio (Due dilligence). Éste es de gran ayuda para los directores corporativos y sirve como un escudo o blindaje ante posibles contra-argumentos, debates, e incluso demandas promovidas durante y después de la transacción. Igualmente lo requieren los vendedores para asegurarse que los compradores tienen todos los elementos para considerar el valor de la transacción. Considerando su importancia, los reportes de Due dilligence deben ser confiados a especialistas de prestigio y con experiencia en la materia.

Para conseguir vender al mejor precio, los empresarios deben preparar la información contable, fiscal y laboral que revele el valor de sus negocios; los compradores deben investigar y evaluar el valor, competencias y sinergias de la empresa objetivo, lo que se facilitará a través de un Due dilligence realizado por profesionales (McCiurkan \& Torres, 2011).

Acoplamiento estratégico de producto-mercado.

En el crecimiento de las fusiones y adquisiciones la estrategia más frecuente utilizada es la diversificación no relacionada, con un $35.8 \%$, en segundo lugar la expansión con un $30.4 \%$, seguida de la integración vertical tanto hacia atrás como hacia delante, con alrededor del $14 \%$ cada uno. Se identifica que las empresas prefieren un mercado doméstico frente al internacional. Estos datos ponen de manifiesto que las A\&F han buscado la integración horizontal o una diversificación no relacionada de las empresas fabricantes para obtener los beneficios derivados de un mayor participación en el mercado, sinergias, la reducción de costos, etc.

La información anterior permite concluir que el acoplamiento estratégico producto- mercado lleva implícito elementos tales como: a) 
identificar el grado de similitud entre los productos y mercados de la empresa a adquirir con la adquiriente, b) analizar la percepción que tiene el consumidor de los productos de la empresa adquirida, c) revisar la compatibilidad de los canales de distribución de ambas empresa, por un lado la adquiriente y por otro la adquirida, d) identificar la posible existencia de confusión de marcas, y e) evaluar si la adquisición acrecentará el servicio al consumidor.

La orientación estratégica de las A\&F se ha estudiado mediante la teoría del ciclo de vida de la empresa (Mueller 1972). En ella se propone que a través del tiempo la empresa nace, se consolida y crece hasta un punto donde es necesario evitar la caída. Esta teoría incorpora la forma en que las A\&F ayudan a evitar esta caída. Las adquisiciones frecuentemente implican un rápido crecimiento de la empresa que conduce a la investigación de Mueller (1972) llamada hipótesis de crecimiento máximo. En esta hipótesis se plantea la hipótesis de que los directivos buscan maximizar el interés personal de su gestión en lugar del bienestar de los accionistas. Estos directivos están más motivados por la expansión de la empresa, que está directamente relacionada con las recompensas no pecuniarias (por ejemplo, el poder), que por la rentabilidad de la empresa, lo cual distorsiona el proceso de A\&F.

Esta teoría propone la importancia del acoplamiento estratégico en el ciclo de vida del producto, y, por lo tanto, en la estrategia de la empresa que compra así se establece la importancia de este factor dentro del proceso de adquisición. Para enfrentar las innumerables complejidades que encierran los mercados, una empresa debe ser eficiente en el desarrollo de nuevos productos, y administrar eficazmente las fases de cambio de gustos y preferencias.

Las empresas pueden obtener nuevos productos a través de dos formas: 1) la adquisición de la totalidad de una empresa y/o adquiriendo una patente 0 una licencia para producir algunos productos, y 2) desarrollar nuevos productos a través de su propio departamento de investigación y desarrollo. Las empresas han optado por la adquisición para facilitar la tarea de generar nuevos productos, debido a que la innovación es costosa y riesgosa. En general buscan afrontar los retos que encierran los diferentes tipos de mercado, a través de la unión con otra empresa que aumente sus fortalezas y disminuya sus debilidades, con la finalidad de penetrar mercado. 
Lo anterior implica: a) identificar el grado de similitud entre los productos y mercados de la empresa a adquirir con la adquiriente, b) analizar la percepción que tiene el consumidor de los productos de la empresa adquirida, c) revisar la compatibilidad de los canales de distribución de ambas empresas: por un lado la adquiriente, y por otro lado la adquirida, d) identificar la posible existencia de confusión de marcas, y e) evaluar si la adquisición acrecentará el servicio al consumidor.

La información anterior nos permite establecer la siguiente definición para el acoplamiento estratégico producto-mercado: en este acoplamiento se busca que la empresa adquirida y la adquiriente se ensamblen, con la finalidad de localizar nuevos mercados identificando sus necesidades existentes, determinar los medios para satisfacer esas necesidades, gestionar los elementos para ofertar esos medios, y difundir la existencia de una propuesta de producto con unos atributos correspondientes a las preferencias y necesidades de los consumidores.

Acoplamiento operativo.

Cada día son más las compañías industriales que descubren que las herramientas de gestión estándar les resultan insuficientes, y empiezan a interesarse por el desarrollo estratégico de sus marcas (Wise, 2009). Esto ayuda a explicar por qué las empresas industriales líderes están reconsiderando la percepción que se tienen de ellas. Buscar que una empresa al adquirir y fusionar se acople lo más rápido y eficientemente posible a la actividad diaria de operación puede significar en muchos casos, el FCE primordial. En la medida que la nueva empresa integra y absorbe los procesos y la tecnología se verá beneficiada más rápido en sus resultados.

Un ejemplo de esto se percibe en las grandes distribuidoras de refrescos, las cuales para obtener un mayor crecimiento, van por franquicias regionales que tienen algo muy valioso: economías de escala. Utilizando como estrategia la fusión de otros competidores de menor tamaño, o apostando por su adquisición, para capitalizar sus economías de escala y aumentar con esto su competitividad.

La teoría de la valoración (Trautwein, 1990) postula que las A\&F son promovidas por directivos que poseen una información superior sobre el valor 
real de la empresa a adquirir que el propio mercado de capitales. La información puede estar vinculada a las ventajas que se derivarían de la adquisición (por ejemplo, sinergias operativas) o también relacionadas con una valoración inferior a la real de la empresa a adquirir. En esta teoría, el comportamiento oportunista de los directivos juega un papel básico.

Es importante considerar también, que de acuerdo a Chase, Aquilano \& Jacobs (2004) la estrategia de operaciones se refiere a la elaboración de políticas y planes para la utilización de los recursos de la empresa en apoyo de la competitividad de la firma a largo plazo.

Es el caso de Coca- Cola Femsa y Arca Contal, estas empresas están utilizando una estrategia de colaboración con embotelladoras regionales con la finalidad de ampliar su penetración de mercado ya que las embotelladoras regionales tienen la virtud de manejar correctamente sus economías de escala y trabajan muy de cerca con los negocios a los que les ofrecen sus productos. En ese contexto, el 19 de septiembre del 2011 Coca-Cola Femsa anunció la adquisición por 11,000 millones de pesos (MDP) de Grupo CIMSA, un embotellador del sistema Coca-Cola que opera en los estados de Morelos y México ( Campa \& Torres, 2011).

En términos de elementos relativos al acoplamiento operativo cabe destacar los siguientes: a) la adaptación local, regional, nacional e internacional de las líneas de producción buscando eficiencia, en los procesos de operación y mantenimiento, b) la facilidad de la transferencia de tecnología en forma oportuna y ordenada, c) el aseguramiento del registro y administración de patentes y derechos de explotación de las mismas, d) la búsqueda de la complementariedad de recursos, e) el logro de economías de integración vertical, y f) la persecución de economías de escala. Algunas de estas variables se observan en la investigación de Chávez (2009).

La investigación de Chávez (2009) plantea la posibilidad de llevar a cabo una A\&F como opción para que las empresas sobrevivan en un mercado cambiante en épocas de economías difíciles. Al respecto, existen los siguientes elementos a destacar: una mayor fuerza de ventas, se puede abarcar un territorio más grande, aumentar la capacidad de servicios, con mayor personal; la capacidad de compras y presión a proveedores; la mayor solvencia económica para poder afrontar los pagos.

La adaptación local, regional, nacional e internacional de las líneas de producción buscando eficiencia en los procesos de operación y 
mantenimiento; la facilidad de la transferencia de tecnología en forma oportuna y ordenada; el registro y administración de patentes y derechos de explotación de las mismas; la complementariedad de recursos, entre otros aspectos, podrían permitirle a la empresa que adquiere, el logro de economías de integración vertical y el logro de economías de escala, debido a que la información que se posee es mejor que la del propio mercado de capitales y con ello se logra una mejor utilización de los recursos de la empresa para apoyar la competitividad de la empresa adquiriente a largo plazo.

Tomando como base la anterior información se propone la siguiente definición de acoplamiento operativo. Este acoplamiento se refiere a la busca del ajuste de los objetivos operativos de la empresa adquirida con la adquiriente, con la finalidad de potencializar los recursos de ambas empresas en conjunto para el logro de la competitividad deseada a largo plazo.

Acoplamiento financiero.

Dado el monto de las inversiones en A\&F, es de esperar que sean fuentes creadoras de valor para las empresas. Sin embargo un estudio del Business Week del 2002 indica que, por el contrario, el 61\% de estas transacciones destruye el valor para los accionistas (Andre, 2004 en Ugalde, 2009).

En la teoría de las creencias optimistas por parte de los inversionistas y los gerentes (Momentum), Rosen (2006) identificó el concepto del impulso Momentum Merger como la correlación que existe entre la reacción del Mercado al anuncio de la fusión y sus condiciones de mercado recientes. Durante el periodo de mayor auge de A\&F existe un mayor grado de optimismo que genera una reacción positiva a todo anuncio de A\&F por lo tanto el precio de las acciones suben más que en otros tiempos, sin embargo este regresa a la normalidad a largo plazo cuando el optimismo se ve remplazado por los resultados.

Una explicación probable a este optimismo, así como a los efectos negativos de los rendimientos a largo plazo de las A\&F se basa en la manera en la que los directivos son recompensados; si estos son recompensados a 
corto plazo por el desempeño de las A\&F, tendrán mayor posibilidad de realizar una adquisición no positiva.

En la Teoría Neoclásica (Momentum) se asume que los administradores actúan para incrementar el valor de los accionistas. En esta teoría, los impulsos Momentum de las A\&F son resultado del incremento en las sinergias de los grupos fusionados. Rosen (2006) encontró evidencia que indica que la reacción a corto plazo al anuncio de la fusión es completamente invertida en el lapso de los siguientes tres años.

$\mathrm{Si}$ los inversionistas esperan una amplia gama de fusiones con creación de sinergias, entonces ellos reaccionaran positivamente cuando se anuncien dichas fusiones. Cuando la expectativa de los inversionistas está basada principalmente en optimismo más que en la realidad, se crea un aumento de interés a corto plazo causado por el anuncio de dicha fusión que a largo plazo será invertida.

Rosen (2006) considera variables tales como el impacto en la relación de pasivo a capital, la oportunidad de ampliar las fuentes de financiamiento, mejorar la cobertura de deuda, tener una expectativa razonable de mejora del valor de la acción, y vigilar los pasivos contingentes.

De igual manera, la teoría de los mercados eficientes, se desarrolla bajo el supuesto que el precio del mercado accionario refleja el valor real de la empresa. Así, puede obtenerse una referencia de valor en la que asumir la hipótesis de la eficiencia de los mercados de capitales, implica aceptar al mismo tiempo que los precios bursátiles de las acciones de una empresa coinciden con los cash flows futuros descontados que ésta genere (Sierra \& Monterrey, 1991).

Para que los ingresos netos mejoren luego de una A\&F, los beneficios obtenidos por la eficiencia en costos, el incremento en los ingresos brutos 0 el valor presente de los flujos de efectivo potenciales de las actividades de innovación, deben ser mayores que la suma de los costos inherentes a la integración, el incremento en la complejidad de los procesos, los costos escondidos de la implementación y el costo propiamente de la combinación. (Zollo \& Singh: 2004, en Ugalde, 2009).

Con base en lo anterior podemos definir el acoplamiento financiero como la búsqueda que hacen las dos empresas, la adquirida y la adquiriente, para que estén funcionando financieramente mejor que antes que se adquiriera la otra empresa. Así, el acoplamiento financiero tiene que ver con 
el impacto en la relación de pasivo a capital, la mejora de la cobertura de deuda y, sobretodo, la expectativa razonable de mejora del valor de la acción.

Acoplamiento de capital humano.

Se han realizado algunos estudios sobre el tema del recurso humano, entre ellos los realizados por Dolz \& Iborra (2005), Pablo (1994), Siehl, Ledford \& Siehl, (1986); sin embargo, cabe destacar los realizados sobre todo a finales de los 90 y principios del nuevo siglo.

La teoría de la ansiedad se ha utilizado debido a que las A\&F típicamente implican cambios a gran escala en la organización; esto puede ser una importante fuente de ansiedad (Seo \& Hill, 2005). Aunque el grado de ansiedad puede variar entre los individuos y dependiendo de las características de la A\&F (Rentsch \& Schneider, 1991); se observa con frecuencia que los empleados experimentan un alto grado de ansiedad cuando se enfrentan a la fusión (Cartwright \& Cooper, 1993). Por otra parte, esta ansiedad puede manifestarse de diferentes maneras para diferentes miembros de la organización en las diferentes etapas del proceso de A\&F.

Seo \& Hill (2005) se centran en la ansiedad experimentada por la amplia base de empleados, una vez que la decisión de A\&F se ha hecho. Antes de la integración se produce la ansiedad de los empleados que tratan de hacer frente a la incertidumbre suponiendo, a menudo el peor escenario posible, para sus futuros puestos de trabajo (por ejemplo, Marks \& Mirvis, 1985; Rentsch \& Schneider, 1991). En particular, la percepción de amenaza de pérdida del empleo puede dar lugar a un aumento de la preocupación con efecto en la productividad.

Existe una tendencia a que los administradores se distraigan con los múltiples detalles de una adquisición, por eso es importante controlar la ansiedad por parte de los empleados al sentir la inseguridad en sus empleos. Dentro de las estrategias para controlar la ansiedad por la pérdida de empleos están: 1) que los directivos hagan un esfuerzo por interactuar más con sus empleados. Los directivos deben ser entrenados en habilidades para escuchar y ayudar a sus empleados a sobrellevar la ansiedad relacionada con A\&F, 2) la práctica del "paracaídas de oro", garantiza que los ejecutivos 
recibirán una indemnización en caso de que pierden su empleo como resultado de las A\&F (Shearer, 2004). La intención de los "paracaídas de oro" es permitir a los ejecutivos analizar objetivamente los beneficios de un acuerdo, a pesar de la probabilidad de que la A\&F podría dejarlos sin trabajo.

La Teoría de la Identidad Social considera que durante el proceso de cambio los empleados van a reaccionar, tratando de alcanzar una posición positiva de su propio grupo en la nueva organización (Haunschild, Moreland, \& Murrell, 1994). La medida en que los empleados estén dispuestos a aceptar una nueva identidad durante una A\&F está relacionada en parte con la situación relativa (por ejemplo, el éxito, presupuesto, etc.) de su grupo actual en comparación con la empresa adquirida, y en el grado en que sean aceptadas las diferencias de condiciones laborales (Terry \& O'Brien, 2001). De acuerdo con Hogg \& Terry (2000); Terry \& O'Brien (2001) los miembros de niveles superiores de la organización pueden reaccionar negativamente a la A\&F porque creen que su posición de superioridad puede estar en riesgo y ven a la A\&F como una amenaza.

El papel de la teoría del rol del conflicto sugiere que la tensión psicológica se produce cuando los individuos están involucrados en varias funciones que son incompatibles (Katz \& Kahn, 1978). La ambigüedad de rol se refiere a la incertidumbre sobre lo que se espera de una persona en una función específica. En las A\&F los procesos de integración implican interrumpir los acuerdos existentes ya sean culturales, estructurales, de empleo y de creación de nuevas disposiciones, lo que resulta en conflicto y ambigüedad de rol.

La teoría de la justicia organizacional busca explicar las decisiones con respecto a volver a seleccionar y desplazar a colaboradores. Las reacciones de los colaboradores a un cambio en la organización, pueden ser influenciadas por tres tipos de percepciones de justicia: 1) la justicia distributiva (Adams, 1965), que es la imparcialidad de los resultados percibidos en comparación con el estándar de justicia de un individuo, 2) el procedimiento de justicia (Thibaut \& Walker, 1975), que es la equidad de los procedimientos utilizados para determinar los resultados, y 3) la justicia interaccional (Tyler \& Bies, 1990), que es cómo son tratados los miembros en la organización por los responsables de determinar los resultados y procedimientos. 
En la A\&F de una empresa intervienen personas de distintos niveles, culturas, capacidades y experiencias que aportan al análisis de la adquisición, a la negociación, y finalmente a la fusión. Las expectativas que tienen los directivos en cuanto a la profundidad de análisis y la visión de futuro son cruciales para prever los posibles conflictos humanos entre las empresas y estipular las interacciones necesarias para que la A\&F sea un éxito.

En términos operativos el acoplamiento de capital humano, implica lo siguiente: a) acoplar a la adquiriente el perfil, características y habilidades del elemento humano de la empresa a adquirir y fusionar, b) crear un equipo multidisciplinario del adquiriente formado por personal interno y personal externo, c) crear un equipo multidisciplinario de ambas empresas, e d) integrar y consolidar el capital humano, las estructuras, los sistemas y los procesos.

En este sentido, el acoplamiento de capital humano implica la evaluación de la gestión eficiente y eficaz de todas las capacidades, actitudes, destrezas y conocimientos de cada una de las personas de ambas empresas, la adquirida y la adquiriente, con la finalidad de optimizar las labores productivas necesarias para que la nueva organización funcione adecuadamente.

Aseguramiento legal y contable.

En nuestro país como a nivel mundial se aprecia una fuerte tendencia a que las empresas se unan y fusionen para competir en mejor forma. En el caso de nuestro país, en el mercado bancario se encuentra la fusión de Banorte con Ixe, en el sector cervecero está FEMSA - Heineken, etc. En el mercado internacional, en el área de Tecnología, se han producido las integraciones más interesantes, como por ejemplo la alianza de AOL con Time Warner y la de Yahoo! con otras empresas del rubro (Lizana \& Pavio, 2002).

Estos procesos se relacionan con elementos contables de la empresa, así, se encuentra lo siguiente: "La información contable tiene tres funciones distintas 1) la presentación de información a usuarios externos, que se interesan en tomar decisiones de inversión y de crédito; 2) la estimación del 
costo de los productos elaborados y de los servicios prestados por la organización; y 3) el suministro de información útil para los administradores internos, que son responsables de la planeación, del control, de la toma de decisiones y de la evaluación del desempeño" (Barfield, Raiborn \& Kinney, 2005, p.4).

Dentro de las perspectivas teóricas para el estudio de este tema se encuentra la siguiente: "la teoría de la contabilidad positiva sostiene que la información contable puede tener consecuencias económicas para las empresas políticamente visibles, ya que los datos contables se emplean como variables de referencia para decisiones de los políticos y de otros grupos interesados" (Giner \& Pardo, 2004, p.680).

En este sentido la teoría de la contabilidad positiva establece que la elección del método contable es de suma importancia ya que conlleva los aspectos económicos y financieros que pueden afectar a la decisión de elegir determinado método. Existen dos métodos contables utilizados: el método de la compra 0 de la adquisición (purchase method o purchase accounting 0 acquisition accounting) y el de la unión de intereses (pooling of interests method 0 pooling method 0 uniting of interests accounting 0 merger accounting) (Giner \& Pardo, 2004).

Las A\&F pueden representar aspectos negativos por lo cual es conveniente que existan disposiciones legales, instituciones legales y especializadas que prevengan situaciones injustas. Entre estas situaciones se puede mencionar el prevenir que cierta industria se convierte en monopolista, ya que esto generaría un aumento en el precio del producto, entre otros aspectos perjudiciales para la competencia.

En este sentido Trautwein (1990) presenta la teoría del monopolio que plantea que las A\&F se llevan a cabo para mejorar la posición competitiva de las empresas. Las fusiones horizontales pueden ir reduciendo la competencia en un mercado; también las fusiones verticales e incluso las que dan lugar a conglomerados pueden generar situaciones de limitación de la competencia. Existen leyes que previenen el monopolio, por ejemplo en Estados Unidos cuentan con la ley antitrust, y en general las disposiciones contra prácticas restrictivas de la competencia a través de su intervención en esta clase de A\&F. Este enfoque enfatiza que a través de un aumento de poder en el mercado, los directivos de la empresa refuerzan su poder mediante las 
sinergias generadas, por lo que se requiere considerar el efecto que tiene en la A\&F que se lleva a cabo.

Las consideraciones anteriores implican tomar en cuenta los siguientes elementos:

a) Contar con todos los contratos, registros, permisos, que tengan consecuencias legales o contables de todas las áreas pertinentes: es necesario contar con esta información, para poder establecer adecuados contratos de cláusulas a futuro donde se garantice por escrito que todo lo que se describió se encuentre dentro de la empresa en las mismas condiciones en las que se describen, b) corroborar que los sistemas produzcan la información completa y oportuna para la correcta administración, y c) absorber y conjuntar las prácticas contables inmediatamente después de la adquisición de una empresa, debido a los problemas que pueden nacer al momento de querer fusionarse.

Con base en la información anterior se establece la siguiente definición del aseguramiento legal y contable: este aseguramiento básicamente consiste en confirmar la situación legal y contable de la empresa objetivo o la empresa adquirida, identificando los riesgos asociados y las posibles contingencias futuras en dichas materias.

Sinergia producto-mercado.

Berger, Bonime, Goldberg \& White (2004) estudiaron la dinámica de la entrada en el mercado después de las A\&F, utilizando datos de los resultados bancarios. Los resultados sugieren que las A\&F están asociados con un aumento significativo, económicamente y estadísticamente hablando, en la probabilidad de entrada. Los datos sugieren que las A\&F afectan a la participación de mercado de un $10 \%$ a un $20 \%$.

De acuerdo con Lizana \& Pavio (2002) dentro de los aspectos a considerar al momento de analizar los efectos que una fusión podría acarrear dentro de un determinado mercado son: mercado relevante; concentración y segmentación; sustituibilidad del producto; características de la demanda; elasticidad de la demanda; barreras de entrada; expectativas de crecimiento del mercado; justificación comercial para una determinada fusión; aprovechamiento de economías de escala. En relación al comportamiento 
predecible después de la fusión es importante demostrar que una determinada fusión no dejará a la entidad fusionada en una posición de tal predominio dentro del mercado relevante que le haga fácil alterar de manera unilateral el libre juego de la oferta y la demanda dentro del mismo.

Una perspectiva teórica de estudio de este tema es la teoría de la eficiencia (Trautwein, 1990) la cual sostiene que las A\&F se realizan para obtener sinergias de gestión, operativas y financieras. En relación con las sinergias producto-mercado, las habilidades directivas habrán de buscar la maximización de los canales de distribución, y lograr la diferenciación de producto y segmentación de mercado de la empresa fusionada, a través de la inteligencia de mercado para buscar nuevos mercados.

De esta manera, dicha sinergia consiste en lograr la diferenciación de producto y segmentación de mercado de la nueva empresa, acelerar la entrada de nuevos bienes o servicios; usar la inteligencia de mercado para atacar nuevos mercados; superar la incertidumbre; absorber rápidamente los nuevos productos enfatizando la interrelación de las líneas actuales, con el ajuste de la unión de las dos empresas, con el objetivo de satisfacer a los clientes en cuanto a lo que quieren, desean y necesitan del mercado.

Así, la sinergia producto-mercado pretende el ajuste perfecto de la unión de las dos empresas, en una sola, con la finalidad de ganar participación o expansión de mercado, acelerar y desarrollar la entrada de nuevos bienes o servicios a nuevos mercados. Su principal motivación es satisfacer al mayor número de clientes en cuanto a lo que quieren, desean y necesitan del mercado, buscando formas alternas de estar en el momento oportuno, en forma y cantidad de lo que el cliente requiere.

De la información anterior es posible definir la sinergia producto mercado como: el vector de crecimiento que representa en conjunto las unidades de negocio de las dos empresas en cuanto a mayor penetración 0 expansión de mercado, ampliación de mercado, diversificación y desarrollo de nuevos productos. En síntesis implica en términos de elementos: a) maximizar la eficiencia de los canales de distribución, b) lograr la diferenciación de producto y segmentación de mercado de la empresa fusionada, c) usar la inteligencia de mercado para atacar y servir nuevos mercados, y d) absorber rápidamente los nuevos productos enfatizando la interacción con las líneas actuales. 
Sinergia operativa.

Los aspectos operativos representan uno de los efectos clásicos resultantes de las fusiones. Una forma de generar sinergia operativa es a través de una mayor utilización de la capacidad instalada, una base más amplia de reparto de gastos indirectos de fabricación, unos lotes de compra más extensos o como consecuencia de una mayor eficiencia en los procesos compartidos, y compartir habilidades técnicas y/o directivas.

En relación con este aspecto, la teoría de la eficiencia, como hemos mencionada, (Trautwein 1990) sostiene que las A\&F se realizan para obtener sinergias de gestión, operativas y financieras. En cuanto a las sinergias operativas, éstas son las resultantes de aplicar las nuevas tecnologías, de lograr ahorros en costos, de compartir habilidades técnicas y/o directivas, con esto mejorar el servicio de entrega al mercado y, aprender los nuevos procesos. Estas se logran cuando se aprovechan conjuntamente las capacidades en marketing y/o producción.

De acuerdo con Skaer (2004) \& Rapallo (2000), se derivan los siguientes aspectos en el logro de la sinergia operativa:

a) La complementariedad de recursos. De acuerdo con Skaer (2004), una de las mayores fuentes de riqueza generadas por la A\&F, es precisamente la complementariedad de recursos, ya que se obtienen sectores de producción, líneas de distribución y productos adicionales que permiten compartir habilidades técnicas o directivas, ampliar la cobertura de mercado, sirviendo como soporte para una mejora en el servicio al cliente.

b) Economías de integración vertical. Cuando las empresas implicadas en una A\&F operan en distintas fases del proceso productivo para la obtención de un mismo producto final, siendo esta unión orientada bien hacia los clientes, o bien hacia los proveedores, pueden producirse reducciones en los costos de aprovisionamiento o de distribución, al igual que la disminución de los costos de control y de coordinación con otras organizaciones.

c) Economías de escala. Estas sinergias se basan fundamentalmente en los ahorros de costos ligados a la mayor dimensión que tendrá la empresa combinada resultante. De éstas se distinguen básicamente dos tipos: estáticas y dinámicas. 
d) Crecimiento. Permite crecer en el mercado actual o penetrar en otros nuevos.

Tomando como base la información anterior se presenta la siguiente definición de sinergia operativa: en la fusión se busca que la unión de dos empresas dé como resultado una mejor utilización de los equipos, tecnología, procesos, ahorro en costos, generación de economías de escala, economías de integración vertical y complementariedad de recursos. La sinergia operativa incluye elementos tales como: a) aplicar las nuevas tecnologías, b) lograr ahorros en costos, c) compartir habilidades técnicas y/o directivas, d) mejorar el servicio de entrega al mercado y, e) aprender los nuevos procesos. Estos elementos coinciden con los estudiados por Skaer (2004) \& Rapallo (2000).

Sinergia financiera.

Mediante el empleo de fondos excedentes se pueden desarrollar sinergias financieras, Menéndez (2004, p. 177), identifica tres fuentes básicas de sinergias financieras.

En primer lugar, la existencia de economías de escala en la emisión y colocación de acciones en los mercados financieros.

En segundo lugar, se ha argumentado que otra fuente de creación de valor procede de la mayor eficiencia en la asignación de los recursos financieros derivada de la creación de un mercado de capitales interno, que acorta los problemas de información asimétrica existentes entre la empresa y el mercado de capitales (externo) acerca del valor de los activos empresariales y de las oportunidades rentables de inversión.

Para finalizar, en el caso de los conglomerados, las empresas pueden desarrollar su capacidad competitiva mediante la adopción de políticas agresivas de precios respaldadas por operaciones de subsidios cruzados de negocios, que debilitan a los competidores.

En conclusión se dice que existe una sinergia financiera, cuando la unión de dos empresas genera ventajas financieras que no sería posible alcanzar de manera independiente por cada una de las empresas involucradas. 
En tercer lugar, la teoría también es consistente con la evidencia de que las empresas diversificadas son menos valiosas que las empresas centralizadas (Servaes 1996), dado que las fusiones facilitan la inversión en proyectos que en principio son poco rentables y que además serían incapaces de recaudar fondos como entidades autónomas. En el modelo, la diversificación no destruye el valor, sino que es el bajo valor de los activos de la empresa adquirida lo que causa la pérdida de valor en la diversificación.

La teoría de la eficiencia (Trautwein, 1990) sostiene que las A\&F se realizan para obtener diversas sinergias entre otras, las sinergias financieras; éstas son las resultantes de la mejora de las razones financieras. Otra fuente de ventajas es el aumento de fuentes de financiamiento así como los términos del mismo; es decir el acceso a capitales cuyo costo sea menor, incrementando asimismo la capacidad de endeudamiento y aumentando el valor de la empresa.

En el aspecto financiero, las fusiones representan la posibilidad de establecer un impacto positivo en la relación de pasivo a capital, mejorar la cobertura de deuda, y tener una expectativa razonable de mejorar el valor de la acción. Por otro lado, con las adquisiciones habrá posibilidades de obtener una sinergia financiera, debido a que pretenden aumentar del valor de las empresas, al considerar la mejora de las razones financieras y el aumento de las fuentes de financiamiento, y con esto mejorar los términos del mismo.

Así el análisis de las sinergias financieras (Lewellen, 1971) implica el análisis de los siguientes elementos: a) la mejora de las razones financieras, b) el aumento de las fuentes de financiamiento, c) la mejora de los términos de financiamiento, y d) el aumento del valor de la empresa.

Cultura asimilada.

La asimilación de una cultura organizacional diferente ha sido identificada como un factor crítico en el proceso de integración post fusión. (Larsson \& Lubatkin 2001; Nahavandi \& Malekzadeh 1988). Por ejemplo, Cartwright \& Cooper (1993) argumentan que una pre-combinación con la cultura organizacional de la empresa con la cual se va a fusionar, juega un rol determinante en los resultados del proceso de A\&F. 
Sin embargo otras investigaciones de procesos de integración post fusiones han tomado una perspectiva de construcción social (Vaara 2003). Examinando la toma de decisiones post fusión, Vaara (2003) muestra que el proceso de integración no siempre se desdobla de una manera racional; continuamente los directivos toman decisiones basados en su formación previa y experiencia, en sus roles y en la relación entre las compañías fusionadas.

Investigadores de las A\&F han comentado la importancia que los directivos tienen en el proceso de integración y los resultados de la fusión. Por ejemplo, Di Gregorio (2001) argumenta que la atención de los altos directivos hacia los problemas de integración es la clave para el éxito en el proceso de integración. Larsson \& Finkelstein (1999) apuntan que la falta de atención en los problemas de integración puede tener serias repercusiones en el éxito de la fusión.

En un ejemplo empírico sobre la importancia de la atención, Hakanson (1995) encuentra que el grado de inclusión del departamento de investigación y desarrollo en el proceso de integración post fusión afecta el proceso de compartir, transferir y explotar las capacidades técnicas de una compañía en fusiones internacionales. Para entender cómo la atención de los directivos afecta el proceso de integración en una fusión, se tienen que examinar los problemas que los directivos atienden de forma prioritaria, qué factores influencian los patrones de atención y cómo impactan dichos factores en los resultados del proceso de integración.

La teoría de la aculturación, originada en la antropología, considera los cambios en ambos grupos que se producen como resultado del contacto entre los grupos culturales (Berry, 1980). Los investigadores han argumentado que el proceso de aculturación también se aplica en la A\&F, e implica la combinación de diferentes culturas organizacionales (valores, creencias o prácticas que definen una organización) o la imposición de uno sobre el otro (por ejemplo, Nahavandi \& Malekzadeh, 1988).

El proceso de aculturación puede ocurrir de maneras diferentes. Berry (1980) identificó cuatro modos en los que la aculturación se lleva a cabo, los cuales han sido adaptados al contexto de A\&F. Estos son: 1) aculturación (miembros de la organización no conservan su vieja cultura o la sustituyen por una nueva); 2) asimilación (miembros de una organización adoptan la cultura de otro); 3) la separación (los miembros de ambas organizaciones 
mantienen su identidad y su cultural original), y 4) la integración (hay cierto grado de cambio en ambas organizaciones).

Cuando los miembros de las dos organizaciones que se combinan no están de acuerdo en el modo deseado de integración, se produce estrés de aculturación. El estrés de aculturación también será mayor cuando el deseo de los miembros de la organización para mantener su cultura separada sea fuerte y, al mismo tiempo, las fuerzas de la integración de la organización también serán fuertes, creando un choque cultural.

Los choques culturales surgen cuando la gente experimenta por primera vez las diferencias en el trabajo diario (por ejemplo, las diferencias en el estilo de comunicación, planificación, supervisión y dirección, el compromiso, el seguimiento y el trabajo en equipo), y considera su camino como superior al nuevo (Marks \& Mirvis, 1992).

Los empleados deben ser sensibilizados sobre la dinámica de choque de culturas y advertirles de no caer en los modos de pensamiento que conducen al choque de culturas; por ejemplo, evaluar las diferencias en términos de buenas o malas (Marks, 1997).

Otra perspectiva de análisis es la teoría de las características del trabajo, la cual se refiere a los cambios en la percepción del ambiente de trabajo. Una manera para evitar evaluaciones negativas de los cambios en el entorno de trabajo, es rediseñar cuidadosamente puestos de trabajo en la post-fusión y organizar de una manera que mantenga o aumente la satisfacción de los colaboradores en sus puestos de trabajo y el compromiso organizacional. Alentar a los colaboradores para que participen en los procesos de rediseño de puestos de trabajo ha sido recomendado como un enfoque útil para reducir la posible resistencia y mantener una actitud positiva durante la transición (Cameron, Freeman \& Mishra, 1991).

Así, se recomienda guiar a los colaboradores para adaptarse a los cambios de trabajo. Esta teoría implica una relación evidente con la variable de estudio "cultura asimilada", ya que considera la idea de lograr la rápida asimilación de voluntades y expectativas del personal de la empresa adquirida, y que el capital humano de ambas empresas comparta valores y actitudes. En particular, los colaboradores deben entender claramente los cambios que se producirán en su entorno laboral y las razones de los mismos (Newman \& Krzystofiak, 1993). 
La cultura influye en las prácticas organizacionales; por ejemplo, conducta, estilos de liderazgo, y los procedimientos administrativos; por lo que representa una forma de crear valor pero a su vez una forma de destruir valor. En la fusión se requiere asimilar la cultura de dos empresas y convertirla en una sola y ponerla en práctica con todos los miembros de la organización, con la finalidad de mejorar resultados.

De acuerdo a la información revisada con anterioridad en este apartado es posible definir la "cultura asimilada", como aquella que implica el entendimiento y aceptación mutua de las metas y objetivos, lograr la rápida asimilación de voluntades y expectativas del personal de la empresa adquirida, lograr confianza para que ambas partes sean capaz de respetarse y apreciarse, y que el capital humano de ambas empresas comparta valores y actitudes, evitando cualquier choque cultural que se pueda presentar en el proceso de A\&F.

De acuerdo con lo anterior la cultura asimilada, requiere una serie de elementos a considerar tales como: a) el entendimiento y aceptación mutua de las metas y objetivos, b) lograr la rápida asimilación de voluntades y expectativas del personal de la empresa adquirida, c) lograr confianza para que ambas partes sean capaces de respetarse y apreciarse, y d) lograr que el capital humano de ambas empresas comparte valores y actitudes.

\section{Modelo de análisis propuesto.}

Mediante esta revisión conceptual se identifican diversas variables que forman parte de los procesos de adquisición y fusión de empresas. Mediante estos aspectos se propone estudiar la importancia relativa de cada uno de ellos en dichos procesos de A\&F. Esta propuesta de estudio enfatiza la necesidad de clarificar la mencionada importancia. Así, en el aspecto de la fusión, autores como Buono \& Bowditch (1989) identificaron tres niveles de integración: a) financiera, o de bajo nivel; b) estratégica, o de nivel medio; $y$ c) operativa, o de fuerte integración. Sin embargo, con respecto a la fusión 0 integración es necesario conocer con mayor detalle y profundidad la importancia, en este caso, de las 5 variables propuestas para integrar una fusión exitosa.

De igual manera, con respecto al proceso de adquisición, se han identificado, propuesto y utilizado diversas variables; en este estudio 
conceptual se han propuesto 5 conceptos que han sido encontrados en la literatura, aunque ello no implica identificar la importancia relativa de cada uno de ellos. Así, a continuación se presenta el modelo gráfico que incorpora y resume el planteamiento conceptual del presente análisis.

Figura 1.- Modelo propuesto.

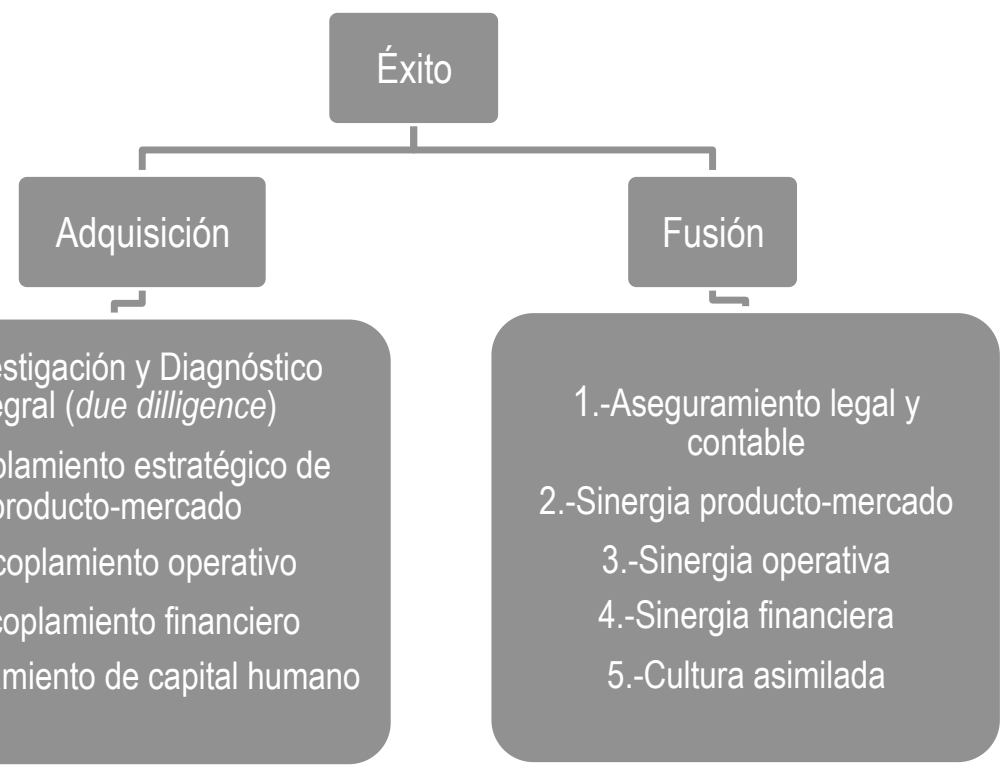

Fuente: Elaboración propia.

\section{Conclusiones.}

En este trabajo conceptual se han planteado dos pregunta de investigación en relación con las variables que pueden utilizarse en el proceso de las A\&F. Una de las preguntas está relacionada con la identificación de los factores clave de un proceso de adquisición exitosa, proponiéndose como tales los siguientes: la investigación y diagnóstico integral (Due dilligence), el acoplamiento producto-mercado, el acoplamiento operativo, el acoplamiento financiero y el acoplamiento de capital humano.

La segunda pregunta también está relacionada con la identificación de la importancia relativa de los factores de un proceso de fusión exitosa, como 
son: el aseguramiento legal y contable, la sinergia producto-mercado, la sinergia operativa, la sinergia financiera y la cultura asimilada.

Vale la pena insistir en que el énfasis de este trabajo conceptual se encuentra en los dos procesos fundamentales relativos a las A\&F, los cuales toman en cuenta las variables que se tienen que tomar en cuenta para una adquisición con éxito, así como las variables que se tienen que tomar en cuenta para una fusión o integración exitosa. Se considera relevante para los efectos de este desarrollo conceptual el enfoque en dichos procesos y sus respectivas variables, aunque no se deja de tener en mente el resultado final de los mismos, que tiene que ver con la creación de valor para la empresa.

Así, el trabajo refleja una revisión de literatura sobre las variables estudiadas, el cual se ha desarrollado con suficiente amplitud. Después de este desarrollo se ha propuesto el modelo gráfico que representa la conclusión de este desarrollo conceptual.

\section{Referencias}

Adams, J. S. (1965). Inequity in social exchange. Advances in experimental social psychology, 2, 267-299.

Anderson, B. \& Ortega, A. (2010). Mi Nuevo FEMSA. Expansión, 1033, disponibe en http://www.cnnexpansion.com/expansion/2010/1033.

Andre, P. (2004) Good adquisitions. CA Magazine, 137(2), 45-58.

Barfield, J. T., Raiborn, C. A., Kinney, M. R., \& Gomez, M. J. (2005). Contabilidad de costos: Tradiciones e innovaciones. Mexico: International Thomson.

Berger, A. N., Bonime, S. D., Goldberg, L. G. \& White, L. J. (2004). The Dynamics of Market Entry: The Effects of Mergers and Acquisitions on Entry in the Banking Industry. The Journal of Business, 77(4), 797-834.

Berry, J. W. (1980). Social and cultural change. In H. C. Triandis \& R.W. Brislin (Eds.), Handbook of crosscultural psychology (Vol. 5, pp. 211-279). Boston: Allyn \& Bacon.

Buono, A. \& Bowditch, J. (1989). The human side of mergers and acquisitions: managing collisions between people and organization, San Francisco: Ed. Joseey-Bass.

Cameron, K., Freeman, S. J.,\&Mishra, A. K. (1991). Practices in white-collar downsizing: Managing contradictions. Academy of Management Executive, 5, 57-73.

Campa, R. \& Torres, J. (2011). A la caza de pequeños imperios, Expansión, 1076, disponibe en http://www.cnnexpansion.com/expansion/2011/1076

Capron, L. (1999). The long term performance of horizontal acquisitions. Strategic Management Journal, 20(11), 987-1018.

Cartwright, S. \& Cooper, C. (1993). The role of culture compatibility in successful organizational marriage. Academy of Management Executive, 7(2), 57-70. 
Chatterjee, S. (1986). Types of synergy and economic value: the impact of acquisitions on merging and rival firms. Strategic Managemenet Journal, 7(2), 119-139.

Chase, R. B., Aquilano, N. J. \& Jacobs, F. R. (2004). Operations management for competitive advantage. Boston, Mass: McGraw-Hill.

Chávez, V. (2009). Los que se van, los que se vienen y los que se quedan. e-semanal, 848, disponible en http://esemanal.mx.

Christensen, C. M., Alton, R., Rising, C. \& Walswck, A. (2011). Why you should pay top dollar for a "killed deal"- and other new rules for making acquisitions. Harvard Business Review, Marzo, 49-57.

De los Ríos, C. V. (2007). Las fusiones y adquisiciones en México en el periodo reciente 1986-2005. Economiaunam, 4(92), 60-92.

DiGeorgio, Richard M. (2001). Making mergers and acquisitions work: What we know and don't know - Part II. Journal of Change Management, 3(3), 259-274.

Dolz, C. \& Ibarra, M. (2005). La integración en las fusiones y adquisiciones: una perspectiva basada en los recursos. Cuadernos de Economía y Dirección de la Empresa, 24, 528.

Feito, I. \& Menéndez, S. (2011). Valuation of the mergers and acquisitions by acquiring shareholders. Universia Business Review, 29, 50-64.

Fluck, Z. \& Lyncj, A. W. (1999). Why Do firms merge and then divest? A theory of financial synergy, The Journal of Business, 72(3), 319-346.

Giner, B. \& Pardo, F. (2004). La elección del método contable en las fusiones empresariales: análisis empírico desde una perspectiva contractual. Revista española de financiación y contabilidad, 23(122), 669-703.

Hakanson, L. (1995). Learning through acquisitions: Management and integration of foreign R\&D laboratories. International Studies of Management and Organization, 25(1-2), 121-157.

Haunschild, P. R., Moreland, R. L. \& Murrell, A. J. (1994). Sources of resistance to mergers between groups. Journal of Applied Social Psychology, 24(3), 1150-1178.

Hernández, P. \& Silva, O. (2011). Fusiones y adquisiciones consolidación de 2010 a 2011. Ejecutivos de finanzas, Septiembre, 50-51.

Hogg, M. A. \& Terry, D. J. (2000). Social identity and self-categorization processes in organizational contexts. Academy of Management Review, 25(1), 121-140.

Hope, M. (2001). Comer es un arte. Expansión, 810, disponibe en http://www.cnnexpansion.com/expansion/2001/810

Hoskisson, R. E., Hitt, M. A. \& Ireland, R. D. (2004). Competing for advantage. Mason, Ohio: South-Western/Thomson Learning.

Ibarra, M. (1997). Las fusiones y adquisiciones como procesos. Dirección y Organización, 18, 78-89.

Jemison, D. B. \& Sitkin, S. (1986). Corporate acquisitions: a process perspective. Academy of Management Review, 11(1), 145-163

Katz, D. \& Kahn, R. (1978). The social psychology of organizations (2nd ed.). New York: John Wiley. 
Ketelhohn, N. \& Marín, J. N (2009). Determinantes de éxito en fusiones y adquisiciones. Incae Business Review, 1(7),16-23.

Larrson, R. \& Finkelstein, S. (1999). Integrating strategic, organizational and human resource perspectives on mergers and acquisitions: A case survey of synergy realization. Organization Science. 10(1), 1-25.

Larsson, R., \& Lubatkin, M. H. (2001). Achieving acculturation in mergers and acquisitions: An international case survey. Human Relations, 54(12), 1573-1607.

Lewellen, A. (1971). A pure financial rationale for the conglomerate merger. Journal of Finance, 26(3), 810.

Lizana, C. \& Pavio, L. (2002). Control preventivo de fusiones y adquisiciones frente a la legislación antimonopolios. Revista Chilena de Derecho, 29(3), 507-541

Luck, S. (1996). Success in Hong Kong. Factors self- reported by successful small business owners. Journal of Small Business, 34(3), 68-75.

Manso, C. F. J. (2003). Diccionario enciclopédico de estrategia empresarial. Madrid: Díaz de Santos.

Marks, M. L. (1997). Consulting in mergers and acquisitions: Interventions spawned by recent trends. Journal of Organizational Change Management, 10(3), 267-279.

Marks, M. L. \& Mirvis, P. H. (1985). Merger syndrome: Stress and uncertainty. Mergers \& Acquisitions, 20, 50-55.

Marks, M. L. \& Mirvis, P. H. (1992). Rebuilding after the merger: Dealing with "survival sickness". Organizational Dynamics, 21(2), 8-32.

Martínez, A. C. (2012). El Poder de engullir empresas. Poder $360^{\circ}$, Marzo, 40-51.

McClurkan T. \& Torres F. (2011). 2011 año de fusiones y adquisiciones, Mundo Ejecutivo, 389.

Menéndez, J. E. (2004). Problemas y Prácticas sobre los Mercados Financieros, Madrid: Díaz Santos.

Morck, R., Schleifer, A. \& Vishny, R. (1990). Do managerial objectives drive bad acquisitions?", Journal of Finance, 45(1), 31-48-

Moyer D. (2004). The Sin of Synergy, Harvard Business Review, 82, 131.

Mueller, D. C. (1972). A Life Cycle Theory of the Firm. The Journal of Industrial Economics, 20(3), 199-219.

Nahavandi, A. \& Malekzadeh, A. R. (1988). Acculturation in mergers and acdquisitions. Academy of Managemenet Review, 13(1), 79-90.

Pablo, A. (1994). Determinants of acquisitions integration level: a decision making perspective. Academy of Management Journal, 37(4). 803-836.

Panasiuk, A. (2004). Los 7 secretos para el exito: Descubrelos para lograr tus suenños. Nashville, TN: Caribe-Betania.

Rapallo, C. (2000). El Euro y el tamaño de las empresas, fusiones y adquisiciones en la Unión Europea. I Simposium Cátedra Jean Monnet de Integración Económica Europea. Madrid.

Ravenscraft, D. J. (1983). Structure-profit relationship at the line of business and indsutry level. Review of Economics and Statistics, 65(1), 22-31. 
Rentsch, J. R. \& Schneider, B. (1991). Expectations for postcombination organizational life: A study of responses to merger and acquisition scenarios. Journal of Applied Social Psychology, 21(3), pp. 233-252.

Robbins, S. P. (1999).Comportamiento organizacional / S.P. Robbins; tr. por Alberto Santiago Fernández Molina. México: Prentice-Hall.

Rosen, R. J. (2006). Merger Momentum and Investor Sentiment: The Stock Market Reaction to Merger Announcements. The Journal of Business, 79(2), 987-1017.

Rovit, S. \& Lemire, C. (2003). Your best M\&A Strategy. Harvard Business Review, 81(3), 1617.

Seo, M. \& Hill, N. S. (2005). Understanding the Human Side of Merger and Acquisition: An Integrative Framework. Journal of Applied Behavioral Science, 41(4), 422-443.

Servaes, H. (1996). The value of diversification during the conglomerate merger wave. Journal of Finance, 51(4), 1201-226.

Siehl, C., Ledford, G. \& Siehl, J. (1986). Strategies for Managing Post-Merger Integration. Paper presentado en el 46 encuentro annual de la Academy of Management, Chicago.

Sierra, G. \& Mty, J. (1991). Análisis contable para fusiones y adquisiciones. Revista Española de Financiación y Contabilidad, 21(69), 933-963.

Sherer, F. \& Ross, D. (1990). Industrial Markets Structure and Economic Performance, 3a.ed, Boston: Houghton Mifflin Company.

Shearer, B. (2004). Taking potshots at golden parachutes. Mergers \& Acquisitions, 39(9), 1418.

Skaer, M. (2004). It's business as usual for Sporlan, Parker. Air Conditioning, Heating \& Refrigeration News, Troy, 223 (9), 6-20.

Terry, D. J. \& O'Brien, A. T. (2001). Status, legitimacy, and ingroup bias in the context of an organizational merger. Group Processes and Intergroup Relations, 4(3), 271-289.

Thibaut, J. \& Walker, L. (1975). Procedural justice. Hillsdale, NJ: Lawrence Erlbaum.

Trautwein, F. (1990). Merger motives and merger prescriptions. Strategic Management Journal, 11(4), 283-295.

Tyler, T. \& Bies, R. (1990). Beyond formal procedures: The interpersonal context of procedural justice. In J. Carroll (Ed.), Applied social psychology and organizational settings (pp. 77-98). Hillsdale, NJ: Lawrence Erlbaum.

Ugalde, N. (2009). Medición de los beneficios netos en las combinaciones de negocios". Ciencias Económicas, 27(2), 301-307.

Vaara, E. (2003) 'Post-acquisition integration as sensemaking: Glimpses of ambiguity, confusion, hypocrisy, and politicization'. Journal of Management Studies, 40(4), 859-894.

Wise, R. (2009). La marca en el ambiente. Gestión de Negocios. 14(2), 22-31.

Wooden, J. R. \& Carty, J. (2006). La pirámide del éxito. Buenos Aires. Miami, Fla: Peniel.

Zollo, M. \& H. Singh. (2004) Deliberate learning in corporate acquisitions: postacquisitions strategies and integration capability in U.S. bank mergers. Strategic Managment Journal, 25(13), 1233-1256. 